Supporting information

\title{
Photosensitizer-Functionalized Mn@Co Magnetic Nanoparticles for MRI/NIR-mediated Photothermal Therapy of Gastric Cancer
}

Zhoufeng Deng ${ }^{\$ a}$, Guanglei Qiao ${ }^{\$ a}$, Lijun $\mathrm{Ma}^{\mathrm{a}^{*}}$, Qian Zhang ${ }^{\mathrm{b}^{*}}$, Ping Zhang ${ }^{\mathrm{c}}$, Daxiang Cui ${ }^{\mathrm{b}^{*}}$

${ }^{a}$ Department of Oncology, Tongren Hospital, Shanghai Jiao Tong University School of Medicine, Shanghai, 200050, China

${ }^{b}$ Department of Instrument Science and Engineering, School of Electronic Information and Electrical Engineering, Shanghai Jiao Tong University, 800 Dongchuan Rd, Shanghai 200240, China.

'Spleen and stomach department, Jiyang District Hospital of Traditional Chinese Medicine, Jinan, Shandong 251400, China

\$Z.F. Deng and G. L. Qiao contributed equally to this paper.

\section{Corresponding Author}

*E-mail: dxcui@sjtu.edu.cn (D.X. Cui), Tel: +86-21-34206375;

qianzhang0130@sjtu.edu.cn (Q.Zhang);

MLJ1971@shtrhospital.com (L.J. Ma).

\section{The calibration curves of ICG}

The calibration curves of ICG were determined by recording the absorption value at $780 \mathrm{~nm}$ with a series of concentrations of ICG in $10 \mathrm{mM}$ DMSO solution via UV-vis spectrophotometer. 

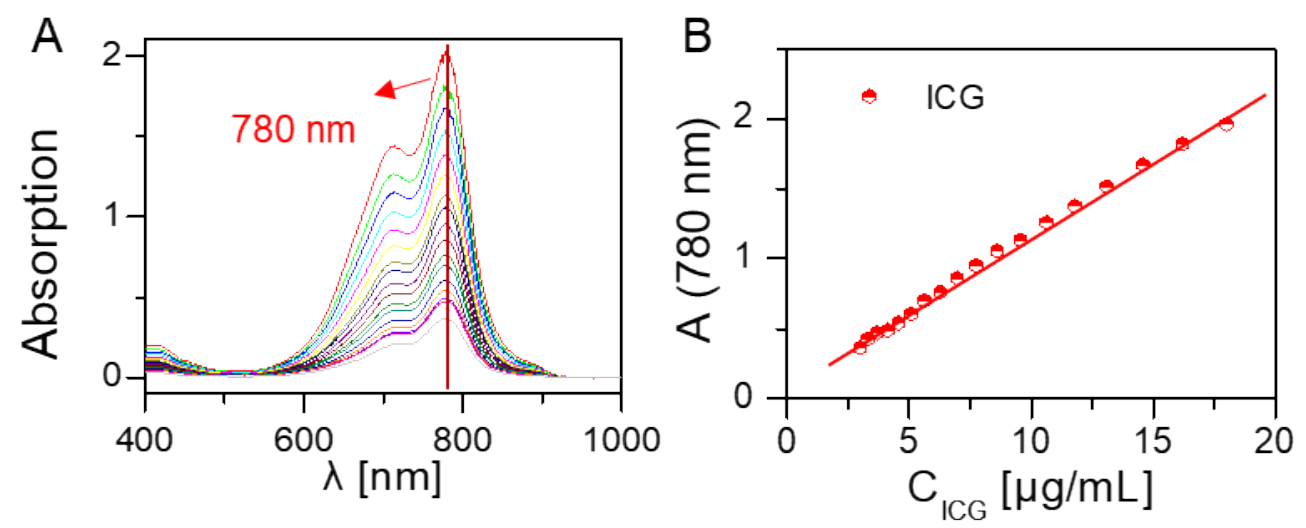

Figure S1. (a) UV-Vis absorption of ICG dispersed in $10 \mathrm{mM}$ DMSO solution with different concentration from 3 to $18 \mu \mathrm{g} / \mathrm{mL}$, and (b) ICG calibration curve calculated from absorption of $780 \mathrm{~nm}$, the acquired equation is $\mathrm{y}=0.112 \mathrm{x}+0.035$.

\section{Cellular ROS detection}

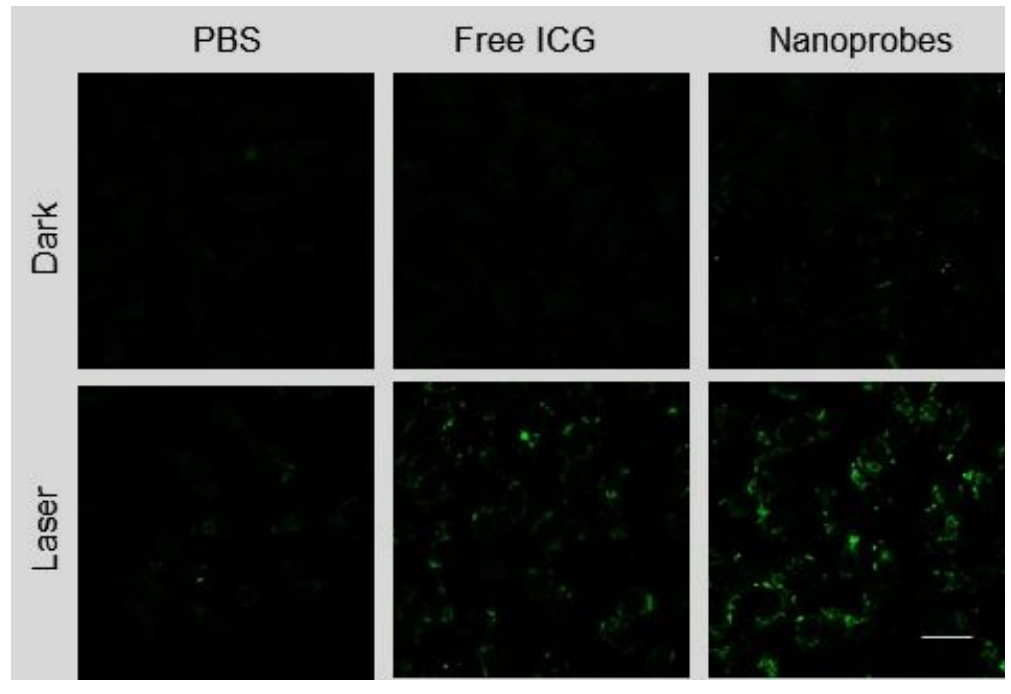

Figure S2. CLSM imaging of MGC-803 cells for cellular ROS detection, after exposure with free ICG and nanoprbes (equivalent ICG dose: $30 \mu \mathrm{g} / \mathrm{mL}$ ) with dark and laser treatment. The carboxy-H2DCFDA dye was used to record the ROS detection (ex: 488nm, em: 510-530 nm). This non-fluorescent molecule can convert to a green-fluorescent form when the acetate groups are removed by intracellular esterase and oxidation (by the activity of ROS) occurs within the 
cell. Scale bar: $20 \mu \mathrm{m}$. 